\title{
Targeting Influencers of Behavior in Nutrition Education
}

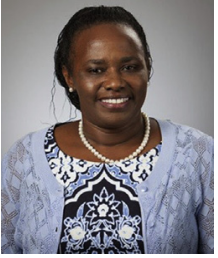

The mission of the Society for Nutrition Education and Behavior (SNEB) is to promote effective nutrition education and healthy behavior through research, policy, and practice. Members of SNEB are engaged as researchers, professors, and industry professionals, as well as with governmental agencies. Our target population includes people from every stage of the lifecycle ranging from pregnancy to the elderly. This wide area of practice affords an extensive range of influence. This editorial will discuss ways of maximizing our impact by targeting the influencers of desired dietary behaviors.

Recently, I took a self-assessment on factors that influence my dietary behaviors and the wide array of influencers was surprising. This list included memories, experiences, people, expectations, fears, geographical locations, and many others. As in my case, persistent behaviors have a myriad of influencers that may include modifiable factors such as awareness, knowledge, and skills. However, influencers may also include unmodifiable factors such as poverty, gender, educational level, age, culture, and geographical location that collectively influence behaviors. ${ }^{1,2}$

The purpose of this editorial is to challenge the current approaches to nutrition education for behavior change. The art and science of influencing healthful behavior has been complicated by the demand for immediate gratification by consumers. Healthy consumers are looking for immediate and quick solutions with minimal effort on their part. ${ }^{3}$ Immediate gratification syndrome focuses on the desired outcome with little or no consideration for the behavior change process or the duration needed to sustainably achieve the preferred outcome. ${ }^{4}$ On the other hand, a well-designed health promotion intervention that produces sustainable and positive changes require time, effort, and a deep understanding of the underlying and direct influencers. ${ }^{5}$ With the understanding that it is not always possible to address all the factors, it is critical to identify and address the influencers with the highest leverage in achieving the desired outcomes. Nutrition educators should be careful not to cave in to the immediate gratification syndrome in an effort to provide quick solutions while designing health related messages for complex issues.

It is, therefore, crucial that as we strive to influence others in adapting healthy lifestyles, we look at what influences the desired behaviors and systematically address those influencers. At the individual level, motivation and ability are important influencers of behavior change according to Fogg's behavior model. ${ }^{6}$ However, it is important to remember that in addition to the individual influencers, the community and societal factors are important influencers of behavior. ${ }^{7}$ For example, a study targeting preschool children was able to achieve its objectives by purposefully including key influencers, such as teachers, coaches, cartoon characters, and videos with children and parents, as part of the intervention. ${ }^{8}$ Similarly, nutrition educators in the community were more likely to be successful when they addressed the environmental, cognitive, and cultural factors after identifying them as critical influencers to behavior change. ${ }^{9,10}$

Given the complexity of behavior change, confounded by the fact that healthful behaviors could be unaffordable or uncomfortable, and that unhealthy choices could be pleasurable and appealing, it is critical for nutrition educators aiming at behavior change to engage every source of influence available, and where possible, to engage the influencers that have the greatest impact to make healthful behavior sustainable. The collective training and experience that SNEB members have acquired in nutrition education positions the organization as a leader and influencer of healthy behavior through research, policy, and practice.

Mary Murimi, PhD, RD SNEB President

\section{REFERENCES}

1. Hofmann W, Baumeister RF, Förster G, Vohs KD. Everyday temptations: an experience sampling study of desire, conflict, and self-control. J Pers Soc Psychol. 2012;102:1318-1335.

2. Newson RS, Lion R, Crawford RJ, et al. Behaviour change for better health: nutrition, hygiene and sustainability. BMC Public Health. 2013;13(Suppl 1):S1.

3. Dassen FC, Houben K, Jansen A. Time orientation and eating behavior: Unhealthy eaters consider immediate consequences, while healthy eaters focus on future health. Appetite. 2015;91:13-19.

4. Schmidt B, Holroyd CB, Debener S, Hewig J. I can't wait! Neural reward signals in impulsive individuals exaggerate the difference between immediate and future rewards. Psychophysiology. 2017;54:409-415.

5. Campbell NC, Murray E, Darbyshire J, et al. Designing and evaluating complex interventions to improve health care. BMJ. 2007;334:455-459.

6. Fogg BJ. A behavior model for persuasive design. Paper presented at: 4th International Conference on Persuasive Technology; April 26-29, 2009; Claremont, CA. http://dl.acm.org/citation.cfm?id =1541999. Accessed May 24, 2017.

7. Golden SD, McLeroy KR, Green LW, Earp JA, Lieberman LD. Upending the social ecological model to guide health promotion efforts toward policy and environmental change. Health Educ Behav. 2015;42(1 Suppl):8S-14S.

8. Hoffman JA, Thompson DR, Franko DL, Power TJ, LeffSS, Stallings VA. Decaying behavioral effects in a randomized, multiyear fruit and vegetable intake intervention. Prev Med. 2011;52:370-375.

9. Hawthorne K, Robles Y, CanningsJohn R, Edwards AG. Culturally appropriate health education for Type 2 diabetes in ethnic minority groups: a systematic and narrative review of randomized controlled trials. Diabet Med. 2010;27:613-623.

10. Nigg CR, Anwar MU, Braun KL, et al. A review of promising multicomponent environmental child obesity prevention intervention strategies by the Children's Healthy Living Program. J Environ Health. 2016;79:18-26. 